\title{
A total synthesis of guanacastepene $\mathrm{C} \dagger$
}

\author{
Goverdhan Mehta, ${ }^{*}$ Kotapalli Pallavi and Jayant D. Umarye \\ Received (in Cambridge, UK) 17th May 2005, Accepted 4th July 2005 \\ First published as an Advance Article on the web 29th July 2005 \\ DOI: 10.1039/b506931a
}

A total synthesis of the structure corresponding to the novel tricyclic diterpene guanacastepene $\mathrm{C}$ has been realized in which a Knoevenagel cyclization serves as a key step to annulate the six-membered C-ring on a stereochemically secured bicyclic hydroazulene precursor.

Guanacastepenes are a structurally diverse and biologically potent group of diterpenoids based on a linearly fused 5,7,6- tricarbocyclic ring system. The first member of this family, guanacastepene A 1 was isolated from an unidentified endophytic fungus growing on the tree Daphnopsis americana in Costa Rica by Clardy et al. ${ }^{1 a}$ in 2000 and its structure was elucidated by X-ray crystallography. Subsequently, several additional guanacastepenes, B-O, have been isolated from the same fungus and structurally characterized, among which guanacastepene $\mathbf{C} \mathbf{2}$ is a close sibling of $\mathbf{1}$ with minor stereo- and functional group variations. ${ }^{1 b, 2}$ Several guanacastepenes, more notably $\mathbf{1}$, have been shown to exhibit impressive activity against methicillin-resistant Staphylococcus aureus (MRSA) and vancomycin-resistant Enterococcus faecalis (VREF). Additionally, guanacastepene A 1 exhibited moderate activity against Gram-positive bacteria, poor activity against Gram-negative bacteria and hemolytic activity against human red blood cells (RBC), thus undermining its therapeutic potential. ${ }^{1 c}$ However, the basic bioactivity attributes of guanacastepenes hold considerable promise and devising means to access their structural variants could be a useful and productive synthetic enterprise. Indeed, guanacastepenes have drawn very widespread attention ${ }^{3}$ from synthetic chemists in the past few years, culminating in the total synthesis ${ }^{4}$ of guanacastepene A 1. Our group has also been in the fray and earlier we reported ${ }^{3 f, j}$ model studies directed towards the synthesis of $\mathbf{1}$ as well as access to several structural analogs ${ }^{3 o}$ of guanacastepenes. Following these early leads and successes, we decided to persist with efforts in the arena and have now accomplished the first total synthesis of guanacastepene C $\mathbf{2}$.

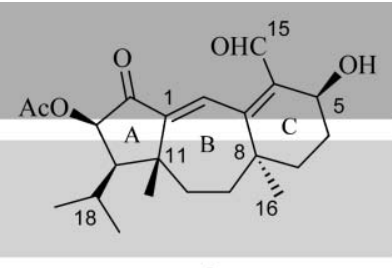

1

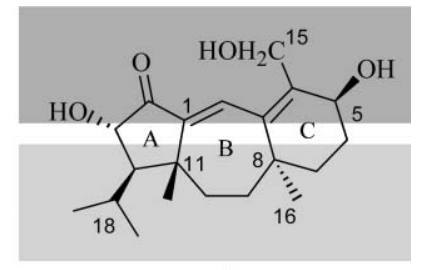

2
Department of Organic Chemistry, Indian Institute of Science,

Bangalore, India.E-mail: gm@orgchem.iisc.ernet.in,

Fax: +91-80-23600283; Tel: +91-80-22932850

$\uparrow$ Electronic supplementary information (ESI) available: ${ }^{1} \mathrm{H}$ NMR and

${ }^{13} \mathrm{C}$ NMR spectral data of selected compounds. See http://dx.doi.org/ $10.1039 / \mathrm{b} 506931 \mathrm{a}$
Guanacastepenes A 1 and C 2 share an identical hydrophobic southern zone (lighter shade) but vary in small but subtle ways in the hydrophilic northern zone (darker shade). More specifically, the $\mathrm{C} 13$ hydroxyl group in $\mathbf{2}$ is free ( $c f$. C13 $\beta$-acetate in 1) and trans disposed with respect to the $\mathrm{C} 12$ isopropyl group, and the $\mathrm{C} 15$ substituent in guanacastepene $\mathrm{C}$ is in the reduced hydroxymethyl form ( $c f$. $\mathrm{C} 15$ aldehyde in 1). In our previous synthetic studies $^{3 f}$ towards guanacastepenes, we had outlined the construction of stereo-defined and adequately functionalized AB-ring segment $\mathbf{5}$, present in these natural products, from the readily available tricyclic enone 3 via $\mathbf{4}$, Scheme 1 . The stereochemical and functionalization pattern present in $\mathbf{5}$ appeared to be more amenable towards the synthesis of guanacastepene C $\mathbf{2}$ rather than guanacastepene A $\mathbf{1}$ and therefore we embarked on a venture targeted towards the former, which through many trials and tribulations has eventuated in the total synthesis of the natural product.

The key issue in the evolution of the AB-ring synthon $\mathbf{5}$ towards the natural product 2 was the annulation of six-membered ring $\mathrm{C}$ and our previous experience had shown the inadequacy of the $\mathrm{RCM}$ and aldol based approaches devised by $\mathrm{us}^{30}$ in delivering the requisite functional and stereochemical array on the annulated ring. Consequently, we opted to explore a Knoevenagel cyclization based protocol for the C-ring annulation on $\mathbf{5}$. LAH reduction of previously described ${ }^{3 o}$ keto ester 5 furnished the diol $\mathbf{6}^{5}$ in modest yield. The two hydroxyl groups in $\mathbf{6}$ needed to be differentiated with the selective protection of the secondary hydroxyl group but, as direct protection manoeuvres were unsuccessful, a more circuitous protocol was devised to deliver $7,,^{5}$ Scheme 2. This required the initial protection of the primary hydroxyl group as the PMB derivative, followed by the protection of the secondary hydroxyl group as the TBS derivative and selective removal of the PMB protection with DDQ. ${ }^{5,6}$ IBX oxidation ${ }^{7}$ of 7 was smooth and led to the aldehyde $\mathbf{8}^{5}$ To set up the Knoevenagel cyclization, bicyclic aldehyde $\mathbf{8}$ was condensed (Wittig-Horner-Emmons reaction) with the dianion derived from ethyl 4-diphenylphosphinoyl-3-oxobutanoate ${ }^{8}$ to append a $5 \mathrm{C}$ fragment and furnish 9 as a mixture of keto-enol tautomers, Scheme 2. Regioselective hydrogenation of $\mathbf{9}$ led to $\mathbf{1 0}$ quantitatively. ${ }^{5}$ TBS deprotection of

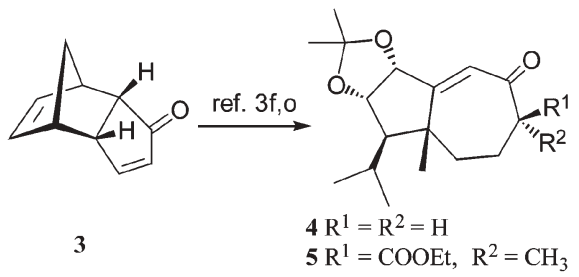

Scheme 1 


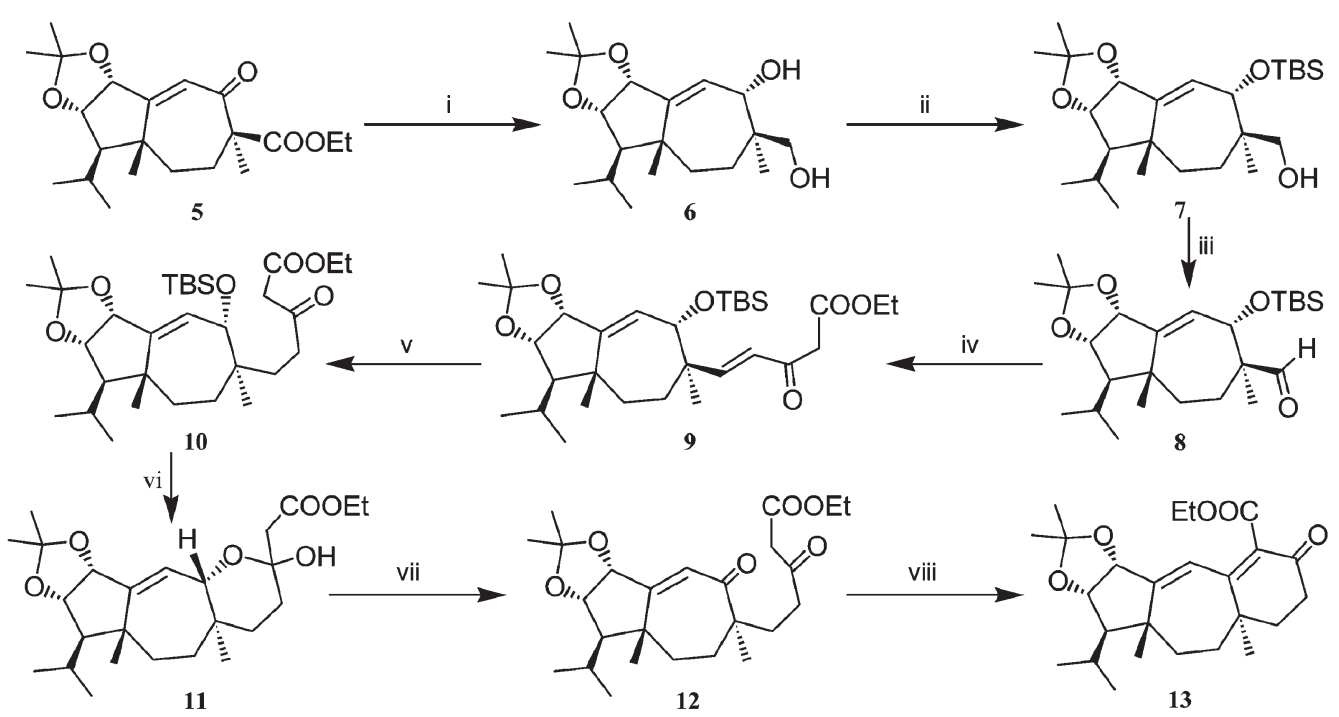

Scheme 2 Reagents and conditions: i, $\mathrm{LiAlH}_{4}$, THF, $0{ }^{\circ} \mathrm{C}$ to rt, $2 \mathrm{~h}, 55 \%$; ii, a. PMBCl, THF, NaH, TBAI, $0{ }^{\circ} \mathrm{C}$ to rt, 5 h, $67 \%$; b. TBSOTf, 2,6-lutidine, DCM, $0{ }^{\circ} \mathrm{C}, 15 \mathrm{~min}, 68 \%$; c. DDQ, DCM- $\mathrm{H}_{2} \mathrm{O}$ (25:1), rt, 2 h, 95\%; iii, IBX, toluene-DMSO (4:1), rt, 3 h, 92\%; iv, $\mathrm{Ph}_{2} \mathrm{POCH}_{2} \mathrm{COCH}_{2} \mathrm{COOEt}, \mathrm{NaH}^{\circ}$, $n \mathrm{BuLi}$, THF, $0{ }^{\circ} \mathrm{C}$ to rt, $6 \mathrm{~h}, 86 \%$; v, $\mathrm{H}_{2}$, EtOAc, $5 \% \mathrm{Pd} / \mathrm{C}(10 \% \mathrm{w} / \mathrm{w}), 30 \mathrm{~min}$, rt, quant.; vi, a. $6 \mathrm{~N} \mathrm{H}_{2} \mathrm{SO}_{4}$, THF-H $\mathrm{H}_{2} \mathrm{O}(4: 1)$, rt, $18 \mathrm{~h}, 80 \%$; b. $2,2-\mathrm{DMP}$, PPTS, rt, 45 min, 91\%; vii, PCC, NaOAc, molecular sieve powder (4 A), DCM, rt, 12 h, $80 \%$; viii, DBU, benzene, $65{ }^{\circ} \mathrm{C}, 12 \mathrm{~h}, 82 \%$.

$\mathbf{1 0}$ led to concomitant acetonide deprotection and intramolecular hemiketalization and the 1,2-diol moiety in the resultant product was reprotected to give 11, Scheme 2. PCC oxidation of $\mathbf{1 1}$ furnished the $\beta$-keto ester precursor $\mathbf{1 2}^{5}$ for the Knoevenagel cyclization. As envisaged, 12, on exposure to DBU, underwent smooth cyclization to generate the C-ring and delivered $13^{5}$ embodying the complete tricarbocyclic framework of guanacastepenes, Scheme 2.

In the dienone ester 13, we had in place the three rings and all the functional elements for further elaboration to the natural product $\mathbf{2}$ and the main task ahead was functional group adjustments. LAH reduction of $\mathbf{1 3}$ led to $\mathbf{1 4}$ as a predominant $\mathrm{C} 5 \alpha-\mathrm{OH}$ isomer (vide infra), albeit in moderate yield, Scheme 3. The diol moiety in $\mathbf{1 4}$ was protected as the diacetate $\mathbf{1 5}$ and acid hydrolysis of the acetonide functionality gave a 4:1 mixture of the diol 17 and the triol 16. Fortunately, triol 16 provided good crystals for X-ray crystal structure determination ${ }^{9}$ and this among<smiles>CC(=O)OCC1=C2C=C3[C@@H]4OC(C)(C)O[C@H]4C(C(C)C)[C@@]3(C)CC[C@]2(C)CC[C@H]1OC(C)=O</smiles>

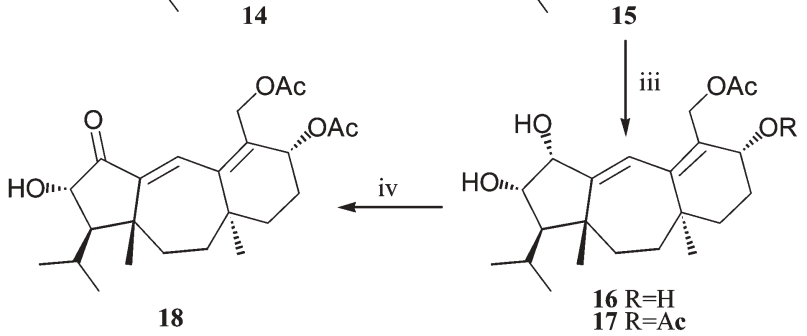

Scheme 3 Reagents and conditions: i, $\mathrm{LiAlH}_{4}, \mathrm{THF},-78{ }^{\circ} \mathrm{C}$ to $\mathrm{rt}, 1 \mathrm{~h}$, $65 \%$; ii, $\mathrm{Ac}_{2} \mathrm{O}, \mathrm{NEt}_{3}$, DMAP, DCM, 5 h, $92 \%$; iii, $4 \mathrm{~N} \mathrm{H}_{2} \mathrm{SO}_{4}, \mathrm{THF}-\mathrm{H}_{2} \mathrm{O}$ (4:1), rt, 8 h, $75 \%$ of $\mathbf{1 7}$ and $20 \%$ of 16; iv, DDQ, THF, $65{ }^{\circ} \mathrm{C}, 6 \mathrm{~h}, 80 \%$. other things revealed the undesired nature of the C5 hydroxyl stereochemistry. DDQ mediated ${ }^{10}$ chemoselective allylic oxidation of $\mathbf{1 7}$ furnished $\mathbf{1 8},{ }^{5}$ formally a 5-epi-guanacastepene $\mathrm{C}$ diacetate, Scheme 3. The elaboration of $\mathbf{1 4}$ to $\mathbf{1 8}$ paved the way for a final assault on the natural product $\mathbf{2}$ and mandated the inversion of $\mathrm{C} 5$ $\alpha-\mathrm{OH}$ stereochemistry.

Consequently, diol 14 was subjected to the standard Mitsunobu protocol to furnish the dibenzoate $\mathbf{1 9}$ in fair yield, Scheme 4. The acetonide protecting group was now disengaged to give $\mathbf{2 0}$ and further chemoselective oxidation ${ }^{10}$ of the allylic hydroxyl group delivered the guanacastepene $\mathrm{C}$ dibenzoate 21, Scheme 4 . At this stage hydrolysis of the benzoate ester groups was expected to deliver the natural product 2. However, for reasons not quite obvious to us, this seemingly straightforward transformation frustrated us to no end and forced us to explore an alternate circuitous route. Thus, the dibenzoate 19 was hydrolyzed to the diol 22 and the hydroxyl groups were reprotected as the diacetate 23, Scheme 4. Deprotection of the acetonide group, even under the most carefully monitored reaction regime, led to some concurrent acetate hydrolysis and a 62:38 mixture of the desired diol diacetate 24 and the triol 25 was obtained. Chemoselective allylic oxidation of $\mathbf{2 4}$ led to the guanacastepene $\mathrm{C}$ diacetate $\mathbf{2 6}$ whose spectral data $^{2}$ was distinctly different from its C5-epimer $18 .^{5}$ Further careful hydrolysis of $\mathbf{2 6}$ delivered a compound corresponding to the reported structure of guanacastepene C 2, Scheme 4. However, as a direct spectral comparison with the natural product was not possible, our arrival at $\mathbf{2}$ has been inferred from the internal consistency of our synthetic sequence and the limited spectral data. $^{11}$

In summary, we have crafted an access to the 5,7,6-fused tricyclic framework of guanacastepene diterpenes from a readily available hydroazulenic precursor employing a Knoevenagel cyclization as the key step and further elaborated this advanced intermediate towards the diterpenoid natural product guanacastepene $\mathrm{C}$. 


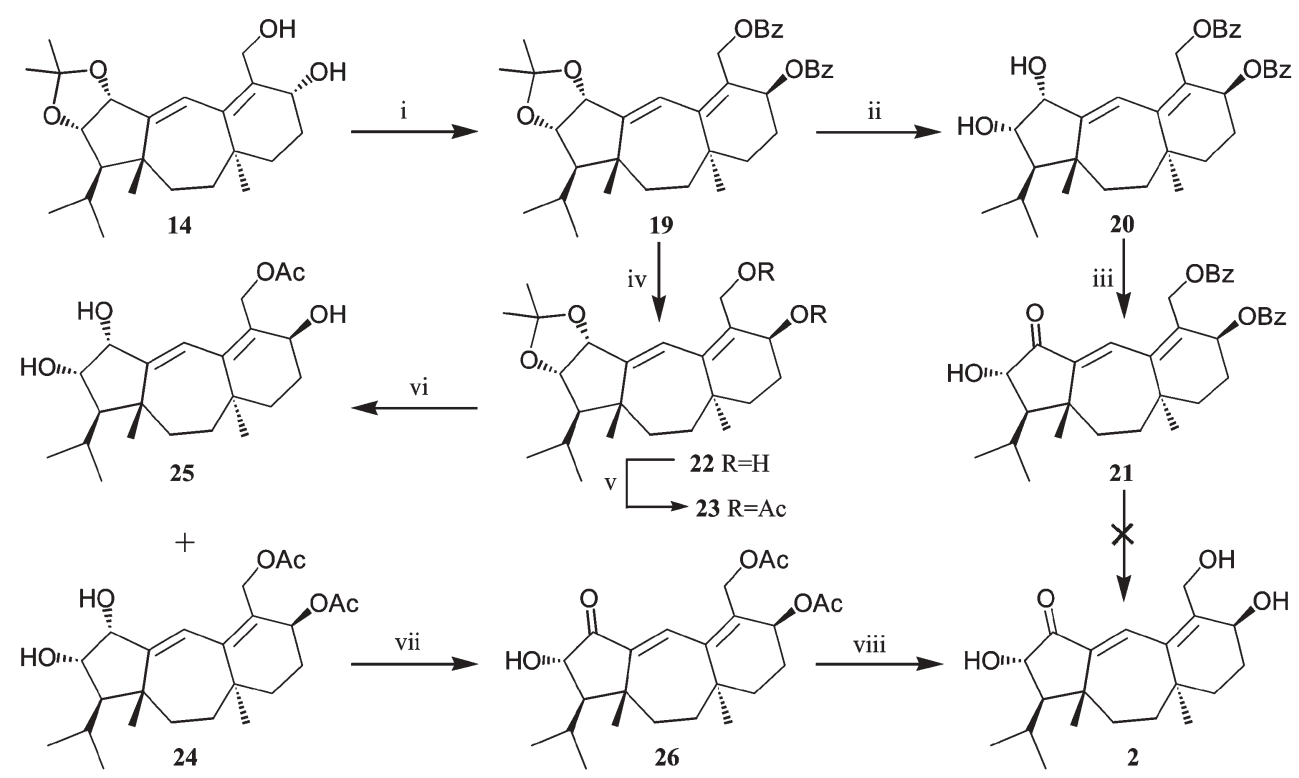

Scheme 4 Reagents and conditions: i, $\mathrm{PPh}_{3}, \mathrm{C}_{6} \mathrm{H}_{5} \mathrm{COOH}, \mathrm{DIAD}, \mathrm{THF},-78^{\circ} \mathrm{C}$ to rt, $2 \mathrm{~h}, 78 \%$; ii, $2 \mathrm{~N} \mathrm{HCl}, \mathrm{THF}, \mathrm{rt}, 6 \mathrm{~h}, 61 \%$; iii, DDQ, THF, $65^{\circ} \mathrm{C}, 6 \mathrm{~h}$, $76 \%$; iv, $\mathrm{LiAlH}_{4}$, THF, $0{ }^{\circ} \mathrm{C}$ to rt, $30 \mathrm{~min}, 84 \%$; v, $\mathrm{Ac}_{2} \mathrm{O}, \mathrm{NEt}_{3}$, DMAP, DCM, rt, $5 \mathrm{~h}, 90 \%$; vi, $4 \mathrm{~N} \mathrm{H}_{2} \mathrm{SO}_{4}$, THF- $\mathrm{H}_{2} \mathrm{O}(4: 1)$, rt, $6 \mathrm{~h}, 44 \%$ of $\mathbf{2 4}$ and $28 \%$ of 25; vii, DDQ, THF, $65{ }^{\circ} \mathrm{C}, 6 \mathrm{~h}, 85 \%$; viii, $\mathrm{K}_{2} \mathrm{CO}_{3}, \mathrm{MeOH}, 0{ }^{\circ} \mathrm{C}$ to $15{ }^{\circ} \mathrm{C}, 2 \mathrm{~h}, 70 \%$.

This research was supported by the Chemical Biology Unit of JNCASR, Bangalore and CSIR (fellowships to KP and JDU), India. We thank SIF and CCD facility at IISc for their help.

\section{Notes and references}

1 (a) S. F. Brady, M. P. Singh, J. E. Janso and J. Clardy, J. Am. Chem. Soc., 2000, 122, 2116; (b) S. F. Brady, S. M. Bondi and J. Clardy, J. Am. Chem. Soc., 2001, 123, 9900; (c) M. P. Singh, J. E. Janso, S. W. Luckman, S. F. Brady, J. Clardy, M. Greenstein and W. M. Maiese, J. Antibiot., 2000, 53, 256.

2 The structure elucidation of guanacastepenes presented a considerable challenge in view of the dynamic conformational equilibrium around the C9- $\mathrm{Cl0}$ bond at the bottom of the seven-membered ring, as a consequence of which the ${ }^{1} \mathrm{H}$ and ${ }^{13} \mathrm{C}$ NMR spectra of these diterpenoids showed an unpredictable number of signals as well as significant variation with solvent and temperature, severely limiting the reliability of spectral data in structure elucidation. Structures in many cases were therefore determined through low temperature crystallization and X-ray diffraction studies ${ }^{1 a, b}$.

3 (a) B. B. Snider and B. Shi, Tetrahedron Lett., 2001, 42, 9123; (b) B. B. Snider and N. A. Hawryluk, Org. Lett., 2001, 3, 569; (c) P. Magnus, M. J. Waring, C. Ollivier and V. Lynch, Tetrahedron Lett., 2001, 42, 4947; (d) G. B. Dudley and S. J. Danishefsky, Org. Lett., 2001, 3, 2399; (e) G. B. Dudley, D. S. Tan, G. Kim, J. M. Tanski and S. J. Danishefsky, Tetrahedron Lett., 2001, 42, 6789; (f) G. Mehta and J. D. Umarye, Org. Lett., 2002, 4, 1063; $(g)$ T. M. Nguyen and D. Lee, Tetrahedron Lett., 2002, 43, 4033; (h) W. D. Shipe and E. J. Sorensen, Org. Lett., 2002, 4, 2063; (i) A. Nakazaki, U. Sharma and M. A. Tius, Org. Lett., 2002, 4, 3363; (j) G. Mehta, J. D. Umarye and V. Gagliardini, Tetrahedron Lett., 2002, 43, 6975; $(k)$ F.-D. Boyer and I. Hanna, Tetrahedron Lett., 2002, 43, 7469; (l) T. M. Nguyen, R. J. Seifert, D. R. Mowrey and D. Lee, Org. Lett., 2002, 4, 3959; (m) P. Magnus and C. Ollivier, Tetrahedron Lett., 2002, 43, 9605; (n) S. N. Gradl, J. J. Kennedy-Smith, J. Kim and D. Trauner, Synlett, 2002, 411; $(o)$ G. Mehta, J. D. Umarye and K. Srinivas, Tetrahedron Lett., 2003, 44, 4233; (p) X. Du, H. V. Chu and O. Kwon, Org. Lett., 2003, 5, 1923; $(q)$ K. M. Brummond and D. Gao, Org. Lett., 2003, 5, 3491; $(r)$ C. C. Hughes, J. J. Kennedy-Smith and D. Trauner, Org. Lett., 2003, 5, 4113; (s) R. Sarpong, J. T. Su and B. M. Stoltz, J. Am. Chem. Soc.,
2003, 125, 13624; $(t)$ A. Srikrishna and D. H. Dethe, Org. Lett., 2004, 6, 165; (u) P. Chiu and S. Li, Org. Lett., 2004, 6, 613; (v) X. Du, H. V. Chu and O. Kwon, Tetrahedron Lett., 2004, 45, 8843; (w) F.-D. Boyer and I. Hanna, J. Org. Chem., 2005, 70, 1077.

4 For the total synthesis, see: (a) D. S. Tan, G. B. Dudley and S. J. Danishefsky, Angew. Chem., Int. Ed., 2002, 41, 2185; (b) S. Lin, G. B. Dudley, D. S. Tan and S. J. Danishefsky, Angew. Chem., Int. Ed, 2002, 41, 2188. For the formal total synthesis, see: (c) B. Shi, N. A. Hawryluk and B. B. Snider, J. Org. Chem., 2003, 68, 1030; (d) F.-D. Boyer, I. Hanna and L. Ricard, Org. Lett., 2004, 6, 1817.

5 All new compounds reported here are racemic and were duly characterized on the basis of spectral and analytical data. As already mentioned $^{2}$ and also observed by others, ${ }^{4 c, d}$ the nature and number of NMR signals in this series of compounds vary due to the presence of conformers. The resonances tabulated here are those observed under our conditions (see ESI + ).

6 Y. Oikawa, T. Yoshioka and O. Yonemitsu, Tetrahedron Lett., 1982, 23, 885.

7 M. Frigerio and M. Santagostino, Tetrahedron Lett., 1994, 43, 8019.

8 A. van der Gen and J. A. M. van den Goorbergh, Tetrahedron Lett., 1980, 21, 3621

9 Crystal data for compound 16: $\mathrm{C}_{22} \mathrm{H}_{34} \mathrm{O}_{5}, M=378.49$, triclinic, space group $P \overline{1}, a=9.1602(16) \AA, b=10.6414(19) \AA, c=12.088(2) \AA$, $\alpha=81.181(3)^{\circ}, \beta=80.027(3)^{\circ}, \gamma=66.679(3)^{\circ}, V=1061.0(3) \AA^{3}, Z=2$, $\rho_{\text {calcd }}=1.185 \mathrm{~g} \mathrm{~cm}^{-3}, F(000)=412, \mu\left(\mathrm{Mo}_{\alpha}-\mathrm{K}_{\alpha}\right)=0.082 \mathrm{~mm}^{-1}, T=$ $293 \mathrm{~K}, R=0.052, R_{\mathrm{w}}=0.122, \mathrm{GOF}=1.083$ for 3067 reflections with $I>2 \sigma(I)$. CCDC 272916. See http://dx.doi.org/10.1039/b506931a for crystallographic data in CIF or other electronic format. An ORTEP diagram with 50\% ellipsoidal probability, has been included in the ESI + .

10 B. A. McKittrick and B. Ganem, J. Org. Chem., 1985, 50, 5897.

11 The original spectra of the natural product were not available from the authors ${ }^{1}$ for direct comparison and full spectral data are not recorded in the communication. ${ }^{1 b}$ We and others have already commented ${ }^{2,4 c, d}$ on the inconsistent nature of the ${ }^{1} \mathrm{H}$ and ${ }^{13} \mathrm{C}$ NMR data in this series of compounds due to dynamic conformational changes. Synthetic $\mathbf{2}$ is no exception in this regard and this makes the independent characterization of $\mathbf{2}$ difficult. However, the comparative ${ }^{1} \mathrm{H}$ NMR data on the $\mathrm{C} 5-\alpha-\mathrm{OH}$ and the $\mathrm{C} 5-\beta-\mathrm{OH}$ series of compounds presented in Schemes 3 and 4, respectively, and the X-ray crystal structure of $\mathbf{1 6}$ clearly point to our acquisition of $\mathbf{2}$. 